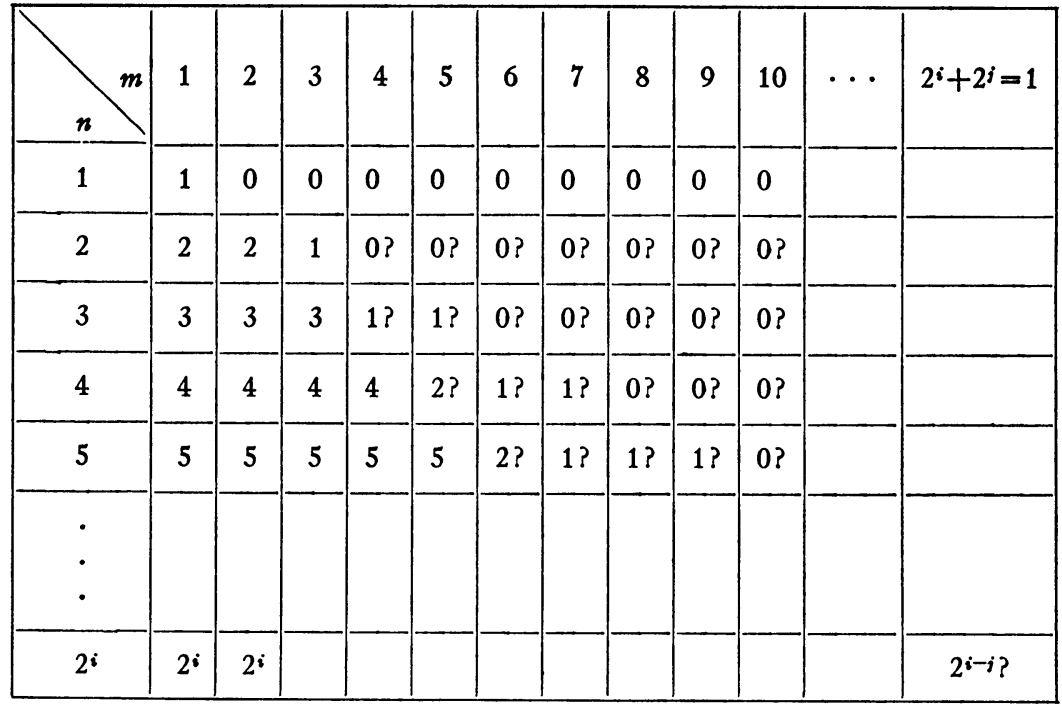

$n \geqq 2$ which raises the dimension of each closed subset of $X$ of positive dimension? Could $f$ be a map of $I^{2}$ into $I^{4}$ ?

If there is such a map, Corollary (3) shows that it is sufficient to consider only the 1-dimensional sets and hence, for compact $X$, only the nondegenerate continua.

\title{
BIBLIOGRAPHY
}

1. W. Hurewicz and H. Wallman, Dimension theory, Princeton, 1948.

UNIVERSITY OF WISCONSIN

\section{COMMENTS ON OPEN HOMOMORPHISMS ${ }^{1}$}

\section{B. J. PETtis}

The present note consists of two comments on the openness of certain homomorphisms, between linear topological spaces (1.t.s.) in the first case and metric groups in the second. Both comments present only refinements on earlier results; the methods, however, are somewhat different and may be of some interest.

Let $\mathfrak{A}$ be any class of closed convex circled sets defined in every

Presented to the Society, April 20, 1951; received by the editors July 15, 1956.

1 This paper was done under Contract N7-ONR-434, Office of Naval Research. 
1.t.s. (e.g., those that are also finite dimensional, or compact, or weakly compact, etc.) and in any l.t.s. $X$ let $\mathfrak{A}(X)$ denote this class. Suppose further that if $X$ is locally convex these are true: (1) the family $\mathfrak{A}(X)^{0}=\left[A^{0}: A \in \mathfrak{A}\right]$, where $A^{0}$ is the set $\left[f: f \in X^{*},|f(a)|<1\right.$ for every $a$ in $A]$ and $X^{*}$ is the adjoint of $X$, forms a local base for a topology, denoted by $\tau\left(\mathfrak{A}(X)^{0}\right)$, with respect to which $X^{*}$ is an 1.t.s., (2) for any closed linear subspace $Y$ of $X$ and any set $B$ in $Y, B$ is in $\mathfrak{A}(Y)$ if and only if $B=A \cap Y$ for some $A$ in $\mathfrak{A}(X)$, and (3) $\left(X^{*}, \tau\left(\mathfrak{A}(X)^{0}\right)\right)^{*}=X$.

Let $Y$ be any closed linear subspace of any locally convex 1.t.s. $X$ and consider $i^{*}$, the adjoint of the injection map of $Y$ into $X$, as a homomorphism of $\left(X^{*}, \tau\left(\mathfrak{A}(X)^{0}\right)\right)$ into $\left(Y^{*}, \tau\left(\mathfrak{A}(Y)^{0}\right)\right)$. Obviously the continuity of $i^{*}$ follows from the "only if" part of (2). The openness is more difficult; it has been shown by Dieudonné [4] when $\mathfrak{A}$ consists of all finite dimensional closed convex circled sets, and by Munroe [12] and Dieudonné [5] when $\mathfrak{A}$ is formed of all compact closed convex circled sets in a Banach space.

Theorem. The homomorphism $i^{*}$ is continuous and open.

The openness will be established if it is shown that for any $A$ in $\mathfrak{A}(X)$ the image set $i^{*}\left(A^{0}\right)$ contains $B^{0}$ for some $B$ in $\mathfrak{A}(Y)$. The obvious choice for $B$ is $A \cap Y$, an element of $\mathfrak{A}(Y)$ by (2). It is then sufficient to show that if $g \in Y^{*}$ and $|g(b)|<1$ for every $b$ in $B$ then $g$ has a continuous linear extension $f$ on $X$ such that $|f(a)|<1$ for every $a$ in $A$. To do this we first recall $[9 ; 1]$ that $A$ is necessarily weakly compact by $(3)$ and the following theorem of Šmulian's $[14 ; 8 ; 3]$ : $a$ weakly closed convex circled set $A$ in an l.t.s. $X$ is weakly compact if and only if it is weakly bounded and given any linear functional $F$ on $X^{*}$ such that $|F(f)| \leqq 1$ whenever sup $[|f(a)|: a \in A] \leqq 1$ there is an element $x_{F}$ in $X$ such that $F(f)=f\left(x_{F}\right)$ for every $f$ in $X^{*}$; when $A$ is weakly compact the element $x_{F}$ can always be chosen in $A$. The following lemma, due to Dieudonné [5] for Banach spaces by a somewhat different method, will obviously complete the proof.

Lemma. In a locally convex l.t.s. $X$ let $A$ be a weakly compact closed convex circled subset and $Y$ a closed linear subspace. Let $g$ be a continuous linear functional on $Y$ such that $|g(b)|<1$ for every $b$ in $A \cap Y$. Then there is a continuous linear extension $f$ of $g$ on $X$ such that $|f(a)|$ $<1$ for every a in $A$.

For each $f$ in $X^{*}$ let $\|f\|=\sup [|f(a)|: a \in A]$, and let $M$ $=[f:\|f\|<1]$. It is clearly sufficient to show that $M$ intersects the set $N$ consisting of all elements of $X^{*}$ that are extensions of $g$. This we 
do by assuming $M$ and $N$ disjoint and applying Mazur's separation theorem $[10,7 ; 3]$ to reach a contradiction. Clearly \|\| is a pseudonorm with respect to which $M$ is a nonvoid open convex set, and $N$ is a linear manifold that is nonvoid by a theorem of Wehausen's $[15 ; 3]$; if they are disjoint Mazur's version of the Minkowski-Ascoli theorem asserts the existence of a linear functional $F$ on $X^{*}$ such that $|F(f)|$ $<1=|F(h)|$ for every $f$ in $M$ and every $h$ in $N$. Applying Smulian's theorem, there is some $x_{F}$ in $A$ such that $F(f)=f\left(x_{F}\right)$ for every $f$ in $X^{*}$. If $x_{F}$ is in $Y$ then on choosing any $h_{0}$ in $N$ we have $\left|F\left(h_{0}\right)\right|=1$ on one hand and $\left|F\left(h_{0}\right)\right|=\left|h_{0}\left(x_{P}\right)\right|=\left|g\left(x_{F}\right)\right|<1$ on the other. Thus $x_{F} \notin Y$. But then, using Wehausen's theorem again, an element $h_{1}$ of $N$ can be chosen that vanishes at $x_{F}$; hence $F\left(h_{1}\right)=h_{1}\left(x_{F}\right)=0$, contrary to $|F(h)|=1$ for $h$ in $N$. Thus $M$ and $N$ must intersect.

Turning now to metric groups, let any open set about the identity element be called a nucleus.

THEOREM. Let $h$ be a homomorphism from a separable complete metric group $X$ into a metric group $Y$, and consider these conditions: (1) $h$ is continuous and $h(U)$ is somewhere dense in $Y$ for each nucleus $U$ in $X,(2) h$ has a closed graph and $h(X)$ is second category in $Y$, (3) $h$ is open and $h(X)$ is second category in $Y$. Then (2) and (3) are equivalent and both are implied by (1).

If (1) holds $h$ obviously has a closed graph, and being a continuous function sending each nonvoid open set into a somewhere dense set it must send second category sets into second category sets $[13, \mathrm{p}$. 298]; thus $h(X)$ is second category in $Y$, so that (2) is verified. To prove (3) in the presence of (2), first observe that if $X$ is a complete metric space and $h$ is a function on $X$ to another metric space and $h$ has a closed graph, then $h$ maps each separable open subset of $X$ into an analytic set and therefore into a Baire set. This theorem, always stated for continuous $h$, as proved in Hausdorff's book [6] needs only the closed graph property for $h$. Thus, in the presence of $(2), h(U)$ is a Baire set for each nucleus $U$ in $X$; the separability of $X$ also implies that $h(U)$ is second category [13, Lemma 2]; since $h$ maps each nucleus into a second category Baire set in $Y$, it is necessarily open $[11 ; 13]$. The proof that (3) implies $(2)$ is in $[13$, p. 303].

CoRollary. If $X$ and $Y$ are separable complete metric groups and $h$ is a homomorphism of $X$ onto $Y$, each of the following implies the other two; $h$ is continuous, $h$ has a closed graph, $h$ is open.

Since $h(X)$ is second category and $X$ is separable, $h(U)$ must be somewhere dense in $Y$ for each nucleus $U$ in $X$. Thus it is sufficient 
to show that if $h$ is open it is continuous. Let $g$ be the natural isomorphism of $Y$ onto $X / K$, where $K$ is the kernel of $h$; since $h$ is open, $g$ is continuous. The group $X / K$ being second category and $Y$ being separable, $g(V)$ must be somewhere dense in $X / K$ for each nucleus $V$ in $Y$. The theorem can now be applied to $g$; since (1) implies (3), $g$ is open, and this in turn implies that $h$ is continuous.

The last theorem and its corollary represent slight improvements on results of Banach [2], Freudenthal, McShane [11], and the present author $[13,3.2,17.1,17.2$, and Theorem 7].

\section{REFERENCES}

1. R. Arens, Duality in linear spaces, Duke Math. J. vol. 14 (1947) pp. 787-794.

2. S. Banach, Über metrische Gruppen, Studia Mathematica vol. 3 (1931) pp. 101-113.

3. N. Bourbaki, Eléments de mathematique, Livre V, Chap. 1-2 (1953), Chap. 3-4 (1955), Paris, Hermann et Cie.

4. J. Dieudonné, La dualité dans les espaces vectoriels topologiques, Ann. École Norm. (3) vol. 59 (1942) pp. 107-139.

5. - Natural homomorphisms in Banach spaces, Proc. Amer. Math. Soc. vol. 1 (1950) pp. 54-59.

6. F. Hausdorff, Mengenlehre, 3d ed. Berlin, Walter de Gruyter, 1935.

7. V. L. Klee, Jr. Convex sets in linear spaces, Duke Math. J. vol. 18 (1951) pp. $443-466$.

8. - Invariant extension of linear functionals, Pacific J. Math. vol. 4 (1954) pp. $37-46$.

9. G. W. Mackey, On convex topological linear spaces, Trans. Amer. Math. Soc. vol. 60 (1946) pp. 519-537.

10. S. Mazur, Über konvexe Mengen in linearen normierten Räumen, Studia Mathematica vol. 4 (1933) pp. 70-84.

11. E. J. McShane, Images of sets satisfying the condition of Baire, Ann. of Math. vol. 51 (1950) pp. 380-386.

12. M. E. Munroe, Homomorphisms on Banach spaces, Bull. Amer. Math. Soc. vol. 54 (1948) pp. 776-781.

13. B. J. Pettis, On continuity and openness of homomophisms in topological groups, Ann. of Math. vol. 52 (1950) pp. 293-308.

14. V. Smulian, Über lineare topologische Räume, Rec. Math. (Mat. Sbornik) N. S. vol. 7 (1940) pp. 425-448.

15. J. V. Wehausen, Transformations in linear topological spaces, Duke Math. J. vol. 4 (1938) pp. 157-169.

\section{TULANE UNIVERSITY}

